\title{
To investigate the effect of ALA and CLA on glucose tolerance and adipose tissue inflammation in the ApoE.3L.CETP transgenic mouse model
}

\author{
Y.M. Lenighan ${ }^{1}$, M.E. O’Reilly ${ }^{1}$, S. Kajani ${ }^{2}$, S.B. Curley ${ }^{2}$, R. Bruen ${ }^{2}$, F.C. McGillicuddy ${ }^{2}$ and \\ H.M. Roche \\ ${ }^{1}$ Nutrigenomics Research Group, Conway Institute of Biomedical and Biomolecular Research and Institute of Food and \\ Health, University College Dublin, Belfield, Dublin, Ireland and \\ ${ }^{2}$ Diabetes Complications Research Centre, UCD Conway Institute of Biomedical and Biomolecular Research and \\ School of Medicine, University College Dublin, Belfield, Dublin, Ireland
}

This abstract was awarded the student prize for best poster original communication.

Alpha-linolenic acid (ALA) and conjugated linoleic acid (CLA) have been associated with anti-inflammatory effects ${ }^{(1,2)}$. A diet high in saturated fatty acids (SFA) induces insulin resistance and a sub-acute, chronic pro-inflammatory state in adipose and the liver ${ }^{(3)}$. This study addressed the hypothesis that supplementing a high SFA-fat diet (HFD) with ALA or CLA (HFD) may ameliorate HFD insulin resistance and attenuate adipose tissue inflammation. ApoE.3L.CETP transgenic mice were fed either a low fat diet (LFD, $10 \%$ kcal from fat), HFD-SFA (45 \% kcal SFA), HFD-ALA (42\% kcal SFA, $3 \% \mathrm{kcal}$ ALA) or HFD-CLA diet (42\% kcal SFA, $3 \% \mathrm{kcal}$ ALA) for 12 weeks. Following the dietary intervention, the metabolic phenotype was characterised using glucose tolerance tests (GTT) and insulin tolerance tests (ITT). Epididymal adipose tissue was harvested to isolate the stromal vascular fraction (SVF) cellular fraction which is enriched in immune cells and stimulated with $1 \mathrm{ng} / \mathrm{ml}$ IL-1 $\beta+\mathrm{TNF} \alpha$ for 18 hours. Resultant adipose IL-6 secretion was measured by ELISA. Prism version 5 (GraphPad Software Inc.) was used for statistical analysis. One- and two-way ANOVA tested for differences between diets in the GTT and adipose IL-6 secretion.

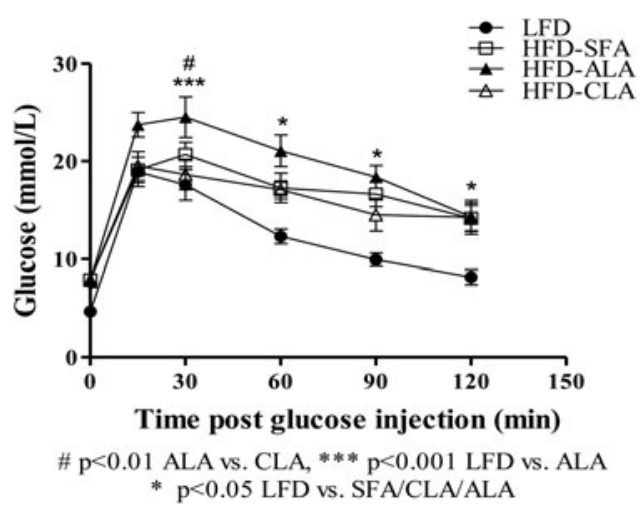

Fig. 1. GTT.

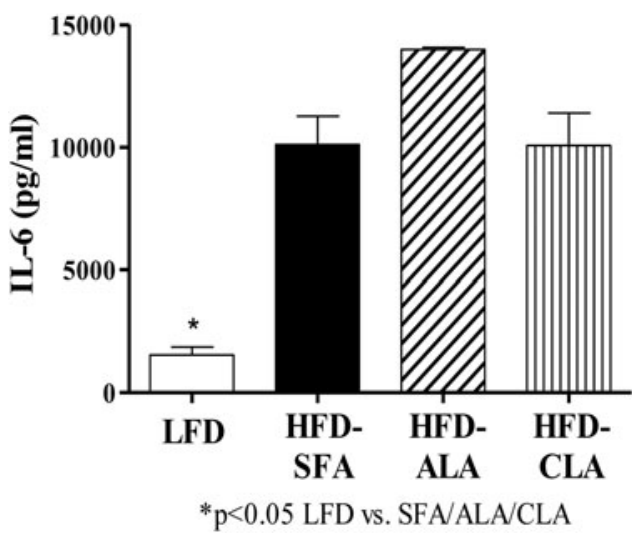

Fig. 2. SVF IL-6 Secretion.

Counterintuitively, Figure 1 demonstrates significantly greater glucose intolerance after the HFD-ALA diet, compared to the HFD-CLA ( $\mathrm{P}<0.01)$. As anticipated the LFD was associated with adequate glucose tolerance compared to all of other HFD groups $(\mathrm{P}<0.05)$ (Figure 1). Adipose SVF IL-6 secretion, in response to the combined IL-1 $\beta+\mathrm{TNF} \alpha$ challenge, demonstrated that the IL-6 response was significantly lower in LFD than all the HFD groups $(\mathrm{P}<0.05)$ (Figure 2.). Fatty acid composition within the different HFDs had no significant differential effect on adipose SVF IL-6. In conclusion, this work demonstrates that substituting dietary SFA with ALA promoted glucose intolerance, and replacement with either ALA or CLA failed to attenuate HFD-induced inflammation in these overweight human transgenic mice. Further analysis is on-going with a view to understanding why HFDs enriched with ALA are potentially more adverse than those enriched in SFA.

This project was funded by the Irish Department of Agriculture, Food and the Marine, 'Healthy Beef' Programme (13/F/514).

1. Zhao G, Etherton TD, Martin KR et al. (2005) Biochem. Biophys. Res. Commun. 336(3) 909-917.

2. Loscher CE, Draper E, Leavy O et al. (2005) J. Immunol. 175(8) 4990-4998.

3. Finucane OM, Lyons CL, Murphy AM et al. (2015) Diabetes 64, 2116-2128. 